\title{
Systematic study of the dolomite (104) surface by bimodal dynamic force microscopy in ultra-high vacuum
}

\author{
Shigeki Kawai ${ }^{1}$, Carlos M Pina ${ }^{2}$, Alexander Bubendorf ${ }^{1}$, Gregor Fessler ${ }^{1}$, \\ Thilo Glatzel ${ }^{1}$, Enrico Gnecco ${ }^{3}$ and Ernst Meyer ${ }^{1}$ \\ ${ }^{1}$ Department of Physics, University of Basel, Klingelbergstrasse 82, 4056 Basel, Switzerland \\ 2 Department of Crystallography and Mineralogy, Complutense University of Madrid, E-28040 \\ Madrid, Spain \\ 3 Instituto Madrileño de Estudios Avanzados, IMDEA Nanociencia, Calle Faraday 9, E-28049 \\ Madrid, Spain E-mail: shigeki.kawai@unibas.ch
}

\begin{abstract}
We have investigated the morphology and structure of dolomite $\mathrm{MgCa}\left(\mathrm{CO}_{3}\right)_{2}(104)$ surfaces by bimodal dynamic force microscopy with flexural and torsional resonance modes in ultra-high vacuum at room temperature. We found that the surface slowly decomposes by degassing $\mathrm{CO}_{2}$ in a vacuum and becomes covered by amorphous clusters, presumably $\mathrm{MgO}$ and $\mathrm{CaO}$. By choosing an optimal sample preparation procedure (i.e. cleaving in a vacuum and mild annealing for stabilizing clusters for a short time), atomically clean surfaces were obtained. The complex tip-sample interaction, arising from carbonate groups and $\mathrm{Mg}$ and $\mathrm{Ca}$ atoms of the surface, induces a large variety of atomic-scale imaging features.
\end{abstract}

\section{Introduction}

For hundreds of years, mineralogists and geologists have studied minerals to gain knowledge about the functioning of the earth system. Traditionally, the composition of minerals and their bulk crystal structures have been investigated by chemical analytical techniques and x-ray diffraction. Only recently, when surface sensitive techniques became available, have the structure and reactivity of mineral surfaces started to be systematically studied [1] (and references therein). Dolomite $\left[\mathrm{MgCa}\left(\mathrm{CO}_{3}\right)_{2}\right]$ is one of the most common rock-forming minerals in nature, and has been widely investigated in the past. Its crystal structure is trigonal and essentially the same as calcite $\left[\mathrm{CaCO}_{3}\right.$ ] [2], but with $\mathrm{Mg}$ and $\mathrm{Ca}$ atoms alternatively occupying the position of $\mathrm{Ca}$ in calcite ([3] and references therein). This mineral poses a fundamental mineralogical question because, in contrast to calcite, we have not yet succeeded in growing a bulk crystal in laboratories at conditions under which it is supposed to be formed in natural environments. Only at temperatures higher than $100{ }^{\circ} \mathrm{C}$ can dolomite be grown under artificially controlled conditions [4-6]. In nature, dolomite is thought to have been formed in the past from sea water supersaturated with respect to this mineral. Thus, the unexplained crystallization of dolomite in nature is referred to in the literature as the 'dolomite problem' [7]. Several authors have pointed out that the anomalous reactivity of the surfaces of this mineral is relevant to the long-standing 'dolomite problem' (e.g. [8-11]). Therefore, the key to understanding dolomite reactivity is to gain a detailed knowledge of the structure of dolomite surfaces and, in particular, of the possible deviations from the bulk structure [12]. In this context, an investigation of the dolomite (104) face at atomic scale using scanning probe microscopy and mass spectrometry in ultra-high vacuum (UHV) can contribute to a future solution of the 'dolomite problem'.

Since the bulk crystal has an insulating nature, the choice of method to study these surfaces with highresolution in real 
space is frequency-modulation dynamic mode atomic force microscopy (DFM) $[13,14]$. A cantilever with a sharp tip attached to its longitudinal end is oscillated at one of its resonance frequencies. The value of this frequency, shifted by the tip-sample interaction, is tracked with a phase-locked loop circuit [15]. A surface topographic image is taken while keeping a constant frequency shift $\Delta f$ by controlling the tip-sample distance with a proportional-integral controller. Since the first systematic achievement of atomic resolution on Si(111)-7 × 7 in 1995 [16], DFM has become a powerful tool for surface science. Recently, this technique has further advanced by incorporating bimodal/multifrequency modes. Bimodal DFM was originally invented for measurements in air and liquid to reduce the tipsample interaction and consequently improve the spatial resolution in tapping mode $[17,18]$. This concept was subsequently extended to atomic-scale measurements in UHV, where it allows one to avoid intrinsic tip and/or sample instabilities in small amplitude operation [19]. Instead of using the two flexural modes, the torsional mode can also be used [20,21]. In this case, the tip apex oscillates parallel to the sample surface and the frequency shift corresponds to the lateral tip-sample interaction [22, 23]. Since the lateral interaction is only responsive to the site-dependent force, the torsional signal has the highest sensitivity to short-range interactions on a flat surface [20, 21, 24], and consequently atomic-scale imaging is reliably and routinely obtained.

Here, we present a study of dolomite (104) surfaces using bimodal DFM with the second flexural and first torsional modes. The morphology and surface structure were systematically studied. We observed a strong degradation of the sample surface during sample preparation. Quadrupole mass spectroscopy measurements give clear evidence that the surface was being degraded. Indeed, the release of $\mathrm{CO}_{2}$ gas and the subsequent coverage of the surface by amorphous structures, presumably $\mathrm{CaO}$ and $\mathrm{MgO}$, was observed. By carefully selecting the preparation parameters, a clean and atomically ordered surface was obtained, and atomic-scale imaging became possible. Similar to previous measurement on an in-laboratory crystallized calcite (104) surface [25, 26], atomic-scale images of the dolomite (104) facet show a large variety of features. Yet the systematic imaging contrast mainly arises from variations in the interactions between the tip and the carbonate groups on the surface.

\section{Experimental details}

All experiments were performed with our home-built ultrahigh vacuum DFM, operating at room temperature (RT) [27]. The dolomite sample was obtained from Eugui, Navarra, Spain. A rather clean and transparent part of the crystal was carefully selected and cleaved to a size of around $5 \times 5 \times$ $3 \mathrm{~mm}^{3}$. Further, clean surfaces were obtained by cleaving in air and in a preparation chamber with pressure $p<3 \times$ $10^{-10}$ mbar. The surfaces were annealed to stabilize atoms and clusters on the surface and to remove residual charges and/or water layers. Unlike high purity ionic salts (e.g. LiF, $\mathrm{NaCl}$ or $\mathrm{KBr}$ ), the preparation parameters strongly affect the dolomite surface structure in UHV. We used commercially available silicon cantilevers (Nanosensors PPP-NCL, $k_{\mathrm{C}}=33 \mathrm{~N} \mathrm{~m}^{-1}$ ) as force sensors. In order to remove water and contaminants from the surfaces, these were annealed at $120{ }^{\circ} \mathrm{C}$ for several hours. Subsequently, the tip was cleaned by $\mathrm{Ar}^{+}$sputtering. We used bimodal DFM with the second flexural and torsional resonance modes to obtain surface topographies [19-21]. The resonance frequency shifts were demodulated by two sets of digital phase-locked loop (PLL) circuits (Nanonis Dual-OC4). The high effective stiffness $\left(k_{2 n d} \approx 1500 \mathrm{~N} \mathrm{~m}^{-1}\right.$ and $k_{T R} \approx 2000 \mathrm{~N}$ $\mathrm{m}^{-1}$ ) enables us to perform stable small amplitude operation [28, 29]. The temperature of the sample during annealing was measured with a thermocouple attached close to the sample holder.

\section{Results and discussions}

\subsection{DFM imaging of dolomite (104) cleaved in air} and in UHV

Figure 1(a) shows a topographic image of the dolomite (104) surface, which was previously cleaved in air. The surface is macroscopically flat (RMS roughness 205 $\mathrm{pm}$ ) but fully covered by amorphous nanostructures. No clear atomic-steps can be distinguished. This might be due to a degradation or reorganization of the dolomite surface when it remains in contact with humid air for a short time (about $5 \mathrm{~min}$ ). A similar phenomenon was also observed on calcite (104) surfaces [30]. In order to avoid such degradation, the sample was in situ cleaved in UHV. In this case, when the crystal was cleaved, the pressure in the chamber suddenly increased to above $10^{-5} \mathrm{mbar}$ and then recovered to the base pressure of 2 $\times 10^{-10}$ mbar within several minutes. Analysis of the outgas will be described later in the context of figure 3 . Figure 1(b) shows a topographic image of the UHV cleaved surface. In contrast to the sample cleaved in air, atomic steps $(\sim 300 \mathrm{pm})$ are clearly observed. In addition, many clusters are seen along the step edges. The inset image of figure 1(b) shows the same area with improved contrast, revealing smaller clusters on the terraces as well. Some of them are quite mobile at RT as proven by the 'noisy' horizontal stripes along the fast scan direction. The presence of mobile clusters on the surface is a critical issue for atomic resolution on this sample. In order to achieve atomic resolution, the tip apex has to be set closer to the sample surface, increasing the possibility of picking up a cluster, and so that stable imaging conditions are hardly obtained. Furthermore, shallow concavities about $10 \mathrm{~nm}$ wide and less than $50 \mathrm{pm}$ deep were found on the whole surface. These features are indicated with arrows in the inset of figure 1(b) and can be attributed to impurities near the surface, such as $\mathrm{Si}, \mathrm{Mn}$ and $\mathrm{Fe}$, which are commonly detected by energy-dispersive-x-ray spectroscopy (EDX) analysis [3]. Such impurities give rise to charge variations and hence induce modulation of the electrostatic force [31-33] on the surface. Since the tipsample distance is controlled by a constant frequency shift at a constant bias voltage $(0 \mathrm{~V})$, these defects modulate the tip-sample distance and hence are clearly visible in the topography [34, 29]. 

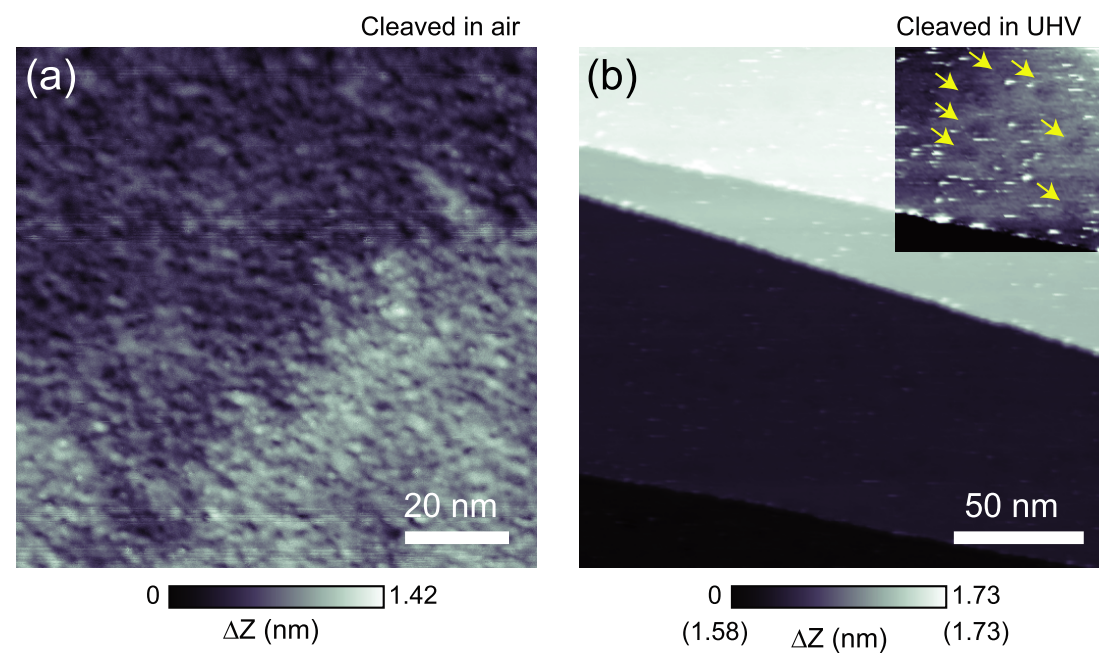

Figure 1. (a) Topographic image of the dolomite (104) surface, obtained by cleaving in air and (b) in UHV. Measurement parameters: (a) $\Delta f_{2 n d}=-15.0 \mathrm{~Hz}, f_{2 n d}=1013730 \mathrm{~Hz}, A_{2 n d}=400 \mathrm{pm}$ and $Q_{2 n d}=11190 ;$ (b) $\Delta f_{2 n d}=-21.0 \mathrm{~Hz}, f_{2 n d}=1019980$ $\mathrm{Hz}, A_{2 \mathrm{nd}}=300 \mathrm{pm}$ and $Q_{2 \mathrm{nd}}=13716$.

@ $80 \operatorname{deg} C$

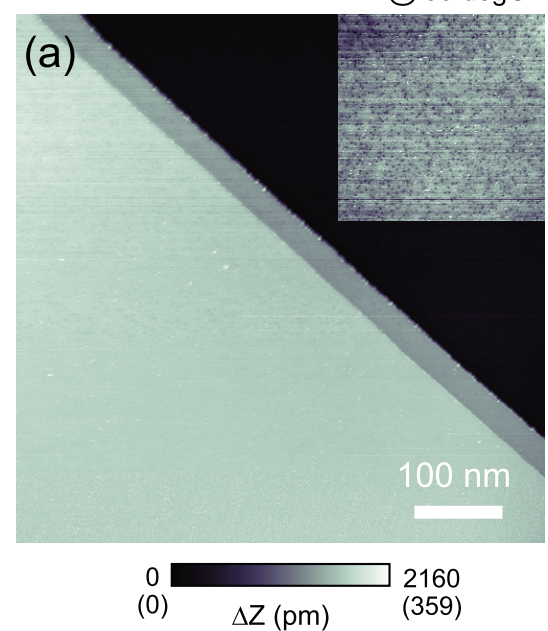

(0) $\Delta \mathrm{Z}(\mathrm{pm}) \quad(359)$
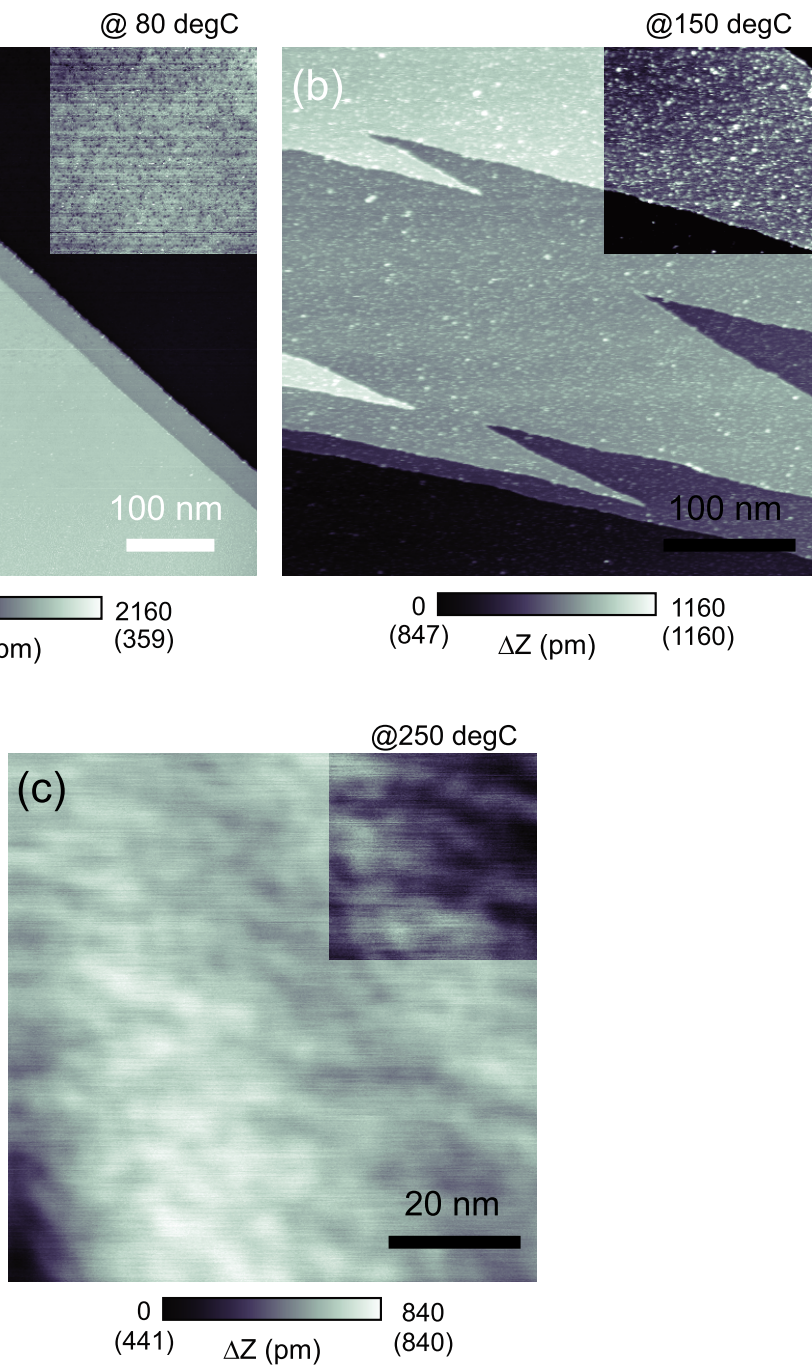

Figure 2. (a) Topographic image of the dolomite sample cleaved in UHV and consequently annealed at $80{ }^{\circ} \mathrm{C},(\mathrm{b})$ at $150{ }^{\circ} \mathrm{C}$, and (c) at $200^{\circ} \mathrm{C}$. Measurement parameters: (a) $\Delta f_{2 n d}=-8.0 \mathrm{~Hz}, f_{2 n d}=1020040 \mathrm{~Hz}, A_{2 n d}=600 \mathrm{pm}$ and $Q_{2 n d}=3226$; (b) $\Delta f_{2 n d}=-10.6 \mathrm{~Hz}, f_{2 n d}=1022070 \mathrm{~Hz}, A_{2 \mathrm{nd}}=400 \mathrm{pm}$ and $Q_{2 \mathrm{nd}}=3308$; (c) $\Delta f_{2 \mathrm{nd}}=-9.0 \mathrm{~Hz}, f_{2 \mathrm{nd}}=1020010 \mathrm{~Hz}, A_{2 \mathrm{nd}}=500 \mathrm{pm}$ and $Q_{2 n d}=13507$. The bias voltage was $0 \mathrm{~V}$. 

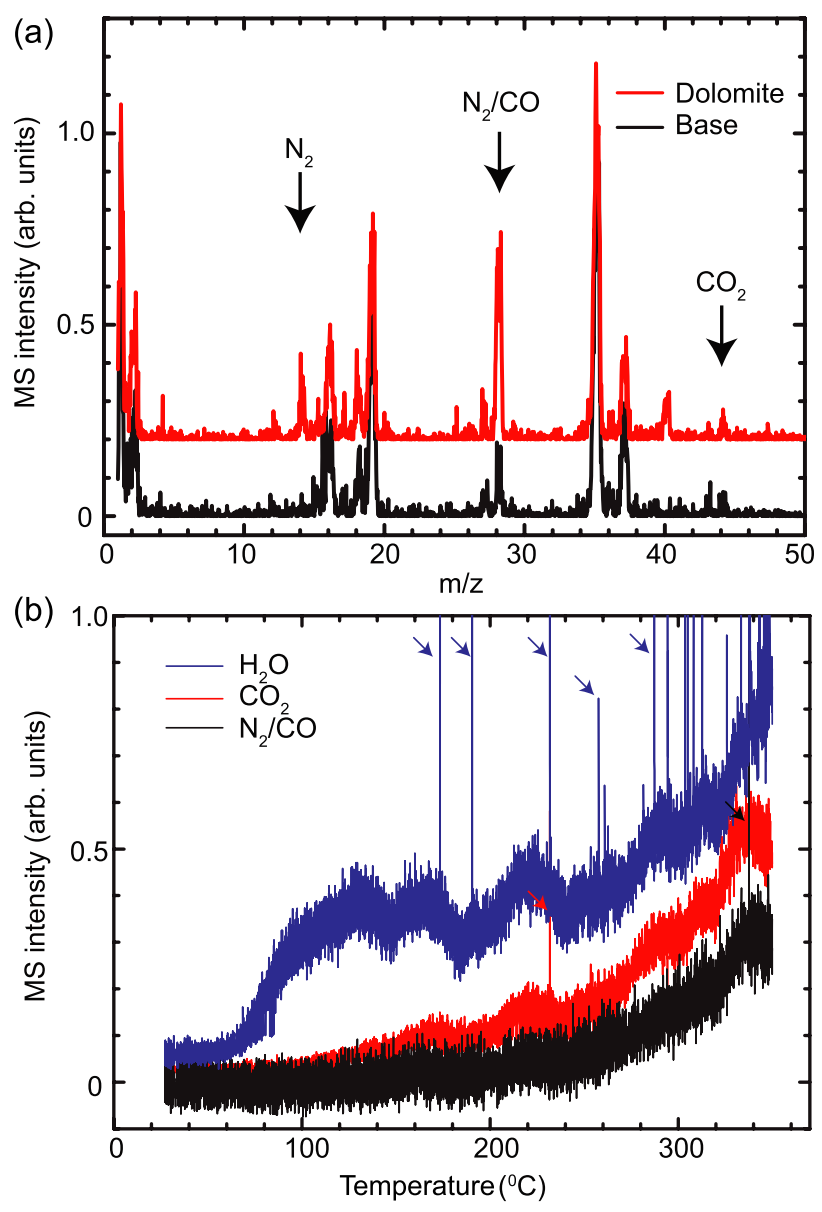

Figure 3. (a) Mass spectrum with and without the dolomite sample in the UHV chamber. The dolomite curve is vertically shifted by 0.2. (b) Thermal desorption spectra of $\mathrm{H}_{2} \mathrm{O}, \mathrm{CO}$ and $\mathrm{CO}_{2}$ in the range of $\mathrm{RT}$ to $350{ }^{\circ} \mathrm{C}$.

In order to stabilize the clusters and improve the resolution, the samples were annealed at three different temperatures. First, the sample was annealed at $80{ }^{\circ} \mathrm{C}$. In this case, the pressure increased to $6 \times 10^{-9} \mathrm{mbar}$, but recovered to $5 \times 10^{-10}$ mbar after several hours. After the sample was cooled down to RT, the surface was imaged with DFM. As shown in figure 2(a), the number of mobile clusters on the terrace was reduced, but the concave features, related to the impurities, remained on the surface, as shown in the inset with improved image contrast. In order to completely remove the clusters, the sample was further annealed at a temperature of $150{ }^{\circ} \mathrm{C}$ for several hours. During annealing, the pressure increased to $2 \times 10^{-8} \mathrm{mbar}$ and recovered to $1 \times 10^{-9} \mathrm{mbar}$. In this case a large number of small clusters were observed, but they were no longer mobile at RT (figure 2(b)). However, when the tip distance was set closer to the sample, the clusters were often picked up, which once again made high-resolution imaging quite challenging. Thus, the sample was further annealed at a temperature of $250{ }^{\circ} \mathrm{C}$. This time the pressure remained fluctuating in the range of $10^{-8}-10^{-7}$ mbar during annealing, and no significant sign of pressure recovery was observed within a few hours. Therefore, the annealing was stopped and the surface was again imaged at RT (figure 2(c)).
Interestingly, the small clusters disappeared, but the surface was fully covered by amorphous nanostructures, although its macroscopic flatness was kept. Since such surface degradation was not reported in previous measurements on calcite (104) in UHV [26, 25], these problems seem to indicate that dolomite (104) surfaces are not energetically stable in a vacuum.

\subsection{Thermal desorption and atomically resolved DFM imaging of dolomite (104) cleaved in air}

In order to further investigate the degradation of the dolomite surface, we analyzed the outgas from the sample with a quadrupole mass spectroscope. Figure 3(a) shows the composition of the residual gas in the UHV chamber. Note that this chamber is not the same as that used for the DFM measurements and the base pressure was relatively good $(4 \times$ $10^{-10}$ mbar) but many peaks due to contamination of the chamber were detected. In this case the dolomite sample was cleaved in air and immediately transferred to the mass spectroscope chamber. An increase in the intensity of $\mathrm{N}_{2}(14$ $\mathrm{m} / \mathrm{z}$ and $28 \mathrm{~m} / \mathrm{z}), \mathrm{CO}(28 \mathrm{~m} / \mathrm{z})$ and $\mathrm{CO}_{2}(44 \mathrm{~m} / \mathrm{z})$ peaks, due to the presence of the dolomite sample, is clearly observed. Since no $\mathrm{O}_{2}$ peak $(32 \mathrm{~m} / z)$ was observed, the increase of the $\mathrm{N}_{2}$ is not related to the air contamination during sample transfer. The increase of the $\mathrm{CO}_{2}$ peak becomes stronger by increasing the sample temperature. Figure 3(b) shows the thermal desorption spectra of $\mathrm{H}_{2} \mathrm{O}(18 \mathrm{~m} / \mathrm{z}), \mathrm{CO} / \mathrm{N}_{2}(28 \mathrm{~m} / \mathrm{z})$ and $\mathrm{CO}_{2}(44 \mathrm{~m} / \mathrm{z})$. By increasing the sample temperature at a rate of $3.5{ }^{\circ} \mathrm{C} \mathrm{min}-1$, the volume of the $\mathrm{CO}_{2}$ decomposition gas increased. At high temperature $\left(>150{ }^{\circ} \mathrm{C}\right)$, intermittent sudden increases of pressure $\left(>1 \times 10^{-5}\right.$ mbar $)$ were observed. At the same time, isolated spikes in the $\mathrm{H}_{2} \mathrm{O}$ concentration were detected. Thus, we attribute the pressure variations to water release near the surface. Since the sample was formed in an aqueous environment, the presence of fluid inclusions within the dolomite crystal is not surprising. When the water near the surface starts boiling, it can be suddenly released, degrading the dolomite surface. This occurs even more often with increasing temperature, but no clear critical temperature was observed. Sometimes, the water desorption was accompanied by spikes in the $\mathrm{CO}_{2}$ and $\mathrm{CO} / \mathrm{N}_{2}$ concentration. Since no correlation between the $\mathrm{CO}_{2}$ and $\mathrm{CO} /$ $\mathrm{N}_{2}$ spikes was observed, a possible decomposition of $\mathrm{CO}_{2}$ into $\mathrm{CO}$ at the mass spectroscope can be excluded. Thus, the 28 $\mathrm{m} / z$ peak most probably corresponds to $\mathrm{N}_{2}$ which was stored in the dolomite. It might be related to the formation of the dolomite in nature. Note that the desorption of $\mathrm{CO}_{2}$ has already been reported from different thermal analysis measurements [35-38]. In these bulk measurements, the decomposition of the crystal was measured by the change in the weight. It is known that the atmosphere plays a role in the chemical decomposition steps. Previous theoretical and experimental works support a mechanism for dolomite decomposition in a vacuum described by the following simple congruent dissociation reaction: $\mathrm{MgCa}\left(\mathrm{CO}_{3}\right)_{2} \rightarrow \mathrm{MgO}+$ $\mathrm{CaO}+2 \mathrm{CO}_{2} \uparrow$ [39-41]. In addition, Powell and Searcy reported the formation of poorly crystalline $\mathrm{MgO}$ and $\mathrm{CaO}$ solids after the decomposition of dolomite in a 

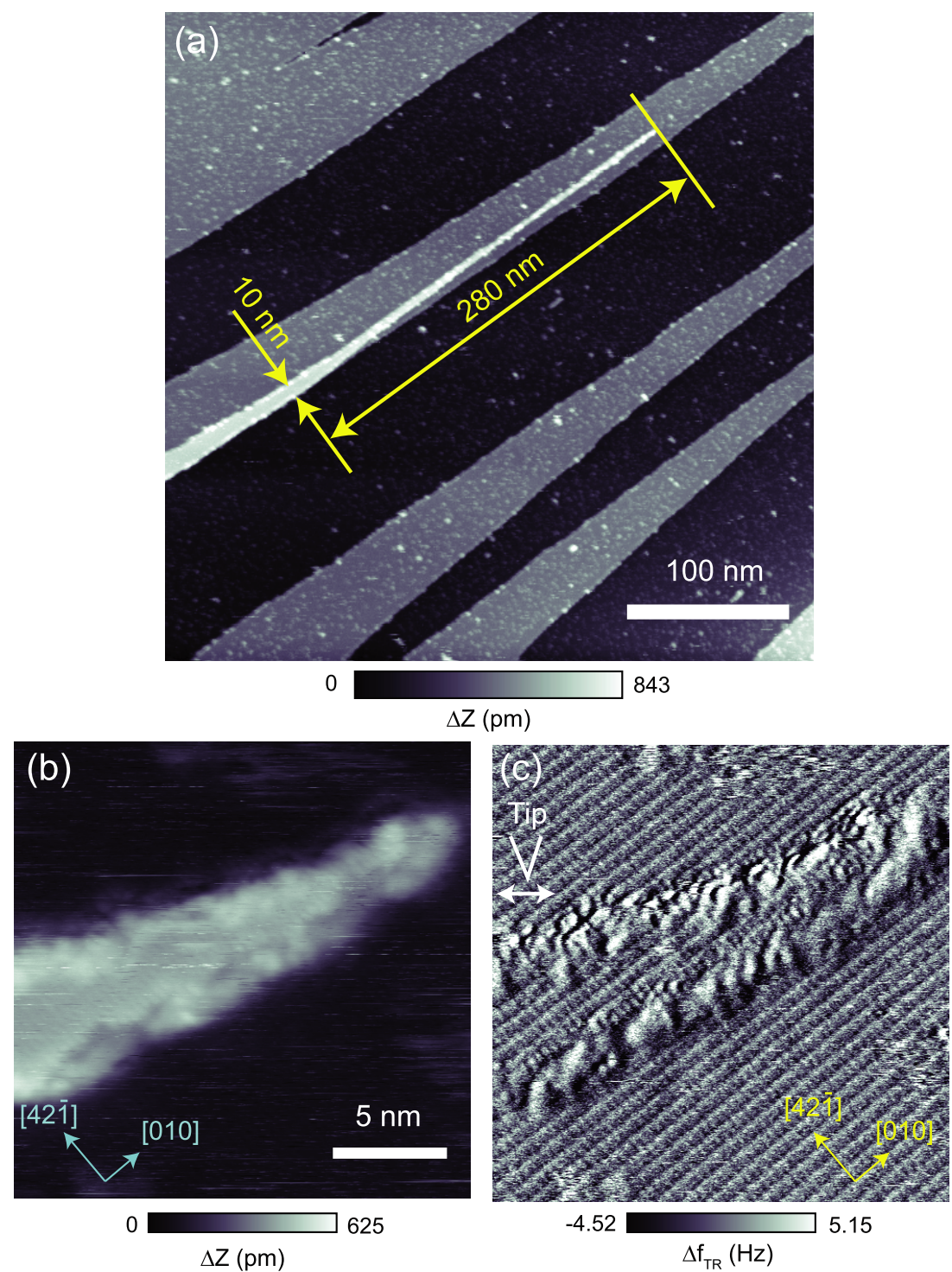

Figure 4. (a) Large-scale topographic image of the dolomite surface, annealed at $150{ }^{\circ} \mathrm{C}$, (b) topographic image, and (c) simultaneously recorded torsional frequency shift $\Delta f_{\mathrm{TR}}$ map in the bimodal detection. Measurement parameters: $(\mathrm{a}) \Delta f_{2 n d}=$ $-16.0 \mathrm{~Hz}$,

$f_{2 n d}=1020030 \mathrm{~Hz}, A_{2 n d}=600 \mathrm{pm}$ and $Q_{2 n d}=3308$; (b), (c) $\Delta f_{2 n d}=-30.0 \mathrm{~Hz}, f_{2 n d}=1020030 \mathrm{~Hz}, A_{2 n d}=400 \mathrm{pm}, Q_{2 n d}=$ $3308, f_{\mathrm{TR}}=1528743 \mathrm{~Hz}, A_{\mathrm{TR}}=10 \mathrm{pm}$ and $\mathrm{Q}_{\mathrm{TR}}=128668$.

vacuum [42]. These results allow us to assume that the clusters observed on our dolomite surfaces are amorphous $\mathrm{MgO}$ and $\mathrm{CaO}$. The reaction in the bulk usually takes place at

$600-900{ }^{\circ} \mathrm{C}$ [35-38]. In contrast, our measurements reveal that the surface decomposition takes place even at RT and is accelerated by increasing temperature.

In order to investigate the process of surface degradation in real space, we imaged the dolomite (104) surface,

annealed at $150{ }^{\circ} \mathrm{C}$ for $4 \mathrm{~h}$. Figure $4(\mathrm{a})$ shows a large-area topograph of the sample. A magnified region around the apex of a wedge-shaped terrace is presented in figure 4(b). The terrace is approximately $300 \mathrm{pm}$ high, meaning that it is terminated by monatomic step edges. These steps are fully covered with clusters. Again, stable imaging conditions were difficult to achieve, and the noise related to tip changes prevented high resolution in the topographic images. However, this was not the case for the torsional frequency shift $f \mathrm{TR}$ maps, which were simultaneously acquired using the bimodal excitation and detection mode (figure 4(c)). Here, atomic-scale contrast was clearly achieved on both upper and lower terraces. This drastic contrast improvement has been also reported on graphite [20] and self-assembled pentacene surfaces [21]. In the present case, the steps run with slight angular offset from the [010] direction, and the number of atomic rows on the upper terrace decreases stepwise down to one. The atomic resolution was lost only at the step edges, where the surface decomposes and $\mathrm{MgO}$ and $\mathrm{CaO}$ reasonably form amorphous clusters.

In order to stabilize the mobile clusters without degradation of the dolomite surface, we annealed the sample at a rather low temperature of $80{ }^{\circ} \mathrm{C}$ for a short time. Figure 5 shows a series of topographic and $\Delta f T R$ images with atomic resolution. A large variety of features were observed. Such a variety is intrinsic to DFM imaging and is due to the distance dependence of $\Delta f$ [43-45], tip-sample 

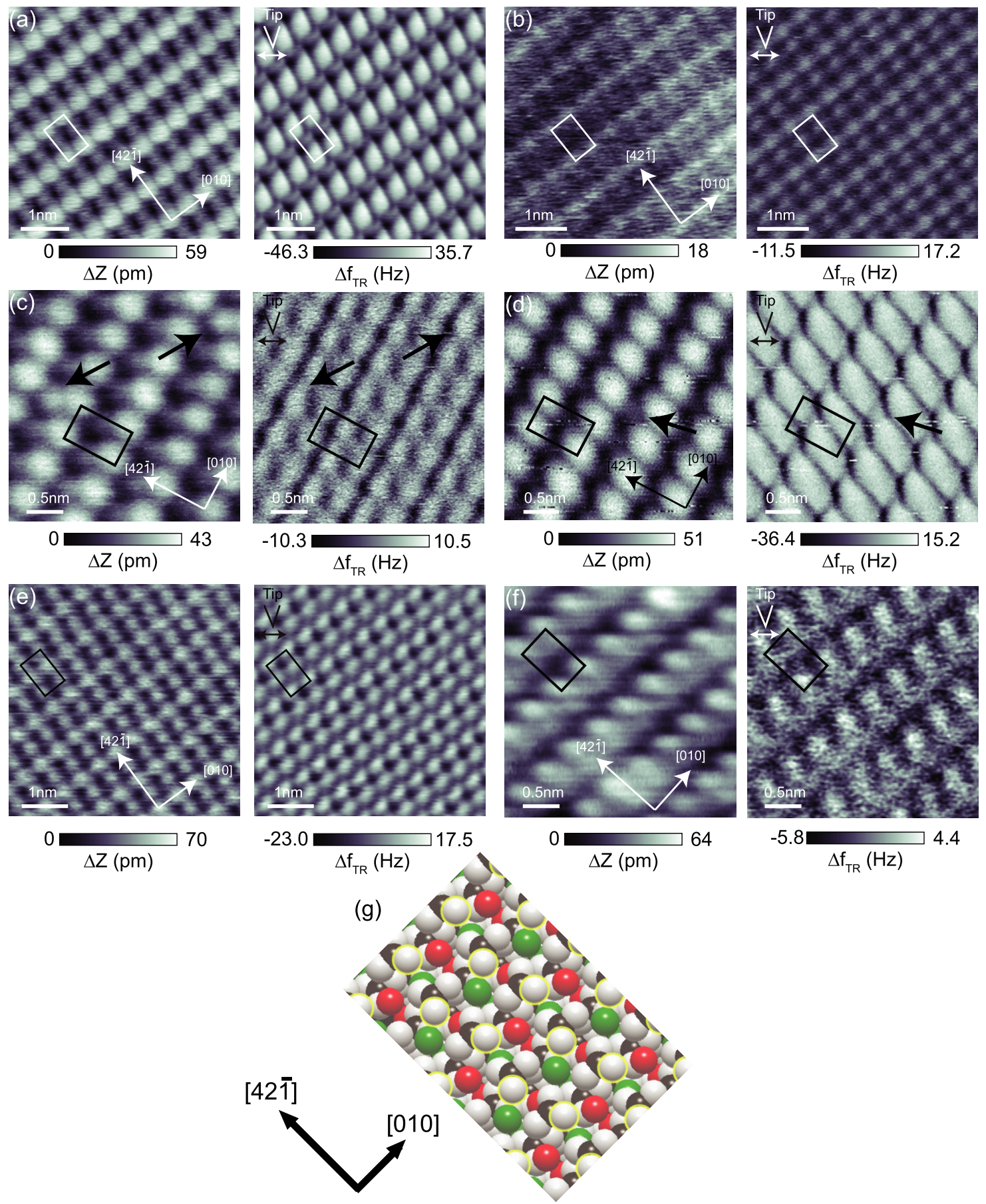

$\Delta \mathrm{f}_{\mathrm{TR}}(\mathrm{Hz})$

Figure 5. (a)-(f) A series of topographic images and $\Delta f_{\mathrm{TR}}$ maps with atomic resolution, obtained with different measurement parameters and tip conditions. (g) Structure of the dolomite (104) surface. Oxygen atoms are represented by white spheres, carbon atoms by black spheres, calcium atoms are red and magnesium atoms are green. Protruding $\mathrm{O}$ atoms are enhanced by yellow circles. Measurement parameters: (a) $\Delta f_{2 n d}=-148.0 \mathrm{~Hz}, f_{2 n d}=1020010 \mathrm{~Hz}, A_{2 n d}=500 \mathrm{pm}, Q_{2 n d}=13507, f_{\mathrm{TR}}=$ $1519453 \mathrm{~Hz}, A_{T R}=10 \mathrm{pm}$ and

$Q_{T R}=145568$; (b) $\Delta f_{2 n d}=-125.0 \mathrm{~Hz}, f_{2 n d}=1020010 \mathrm{~Hz}, A_{2 n d}=500 \mathrm{pm}, Q_{2 n d}=13507, f_{\mathrm{TR}}=1519450 \mathrm{~Hz}, A_{\mathrm{TR}}=10 \mathrm{pm}$ and $Q_{T R}=145568$; (c) $\Delta f_{2 n d}=-31.3 \mathrm{~Hz}, f_{2 n d}=1006000 \mathrm{~Hz}, A_{2 n d}=800 \mathrm{pm}, Q_{2 n d}=13915, f_{\mathrm{TR}}=1537869 \mathrm{~Hz}, A_{\mathrm{TR}}=10 \mathrm{pm}$ and $Q_{T R}=85539 ;$ (d) $\Delta f_{2 n d}=-70 \mathrm{~Hz}, f_{2 n d}=1006001 \mathrm{~Hz}, A_{2 n d}=800 \mathrm{pm}, Q_{2 n d}=13915, f_{\mathrm{TR}}=1537877 \mathrm{~Hz}, A_{\mathrm{TR}}=10 \mathrm{pm}$ and $Q_{\mathrm{TR}}=85539$; (e) $\Delta f_{2 \mathrm{nd}}=-24.9 \mathrm{~Hz}, f_{2 \mathrm{nd}}=1020010 \mathrm{~Hz}, A_{2 \mathrm{nd}}=1.0 \mathrm{~nm}, Q_{2 \mathrm{nd}}=12938, f_{\mathrm{TR}}=1519450 \mathrm{~Hz}, A_{\mathrm{TR}}=20 \mathrm{pm}$ and $Q_{\text {TR }}=145568$; (f) $\Delta f_{2 n d}=-23.0 \mathrm{~Hz}, f_{2 n d}=1006001 \mathrm{~Hz}, A_{2 n d}=800 \mathrm{pm}, Q_{2 \mathrm{nd}}=13915, f_{\mathrm{TR}}=1537877 \mathrm{~Hz}, A_{\mathrm{TR}}=10 \mathrm{pm}$ and $Q_{T R}=85539$.

deformations [46, 47] and the condition of the tip apex $[48,49]$. Although a characterization of the tipsample interaction is particularly interesting for understanding the imaging mechanisms in DFM [5052], here we focus on the analysis of the material only. The unit cell of the (104) surface is indicated by rectangles. Ideally, each unit cell contains one $\mathrm{Mg}$, one $\mathrm{Ca}$ and two $\mathrm{CO}_{3}$ with alternating orientations (see figure $5(\mathrm{~g})$ ). In most cases, spots with two different sizes 
(a)
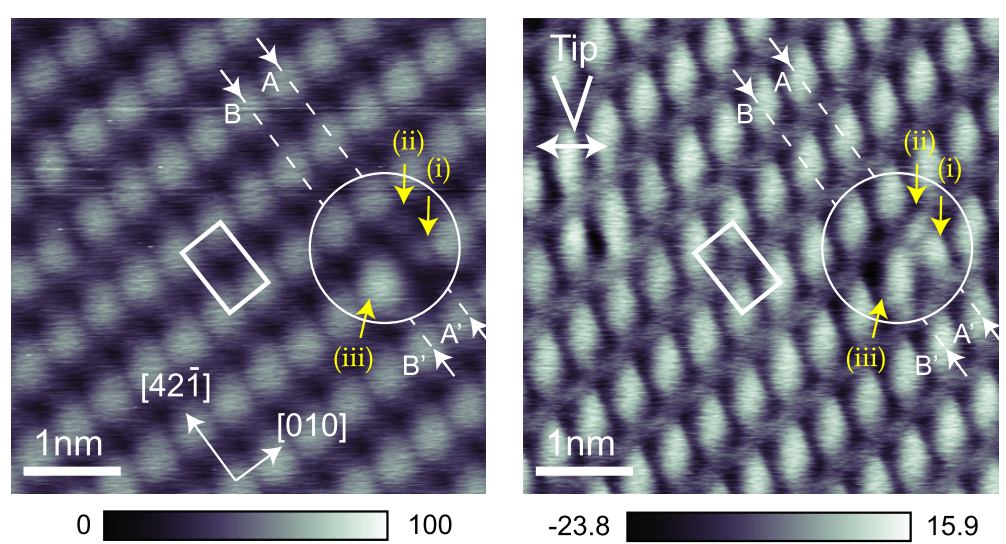

(b)
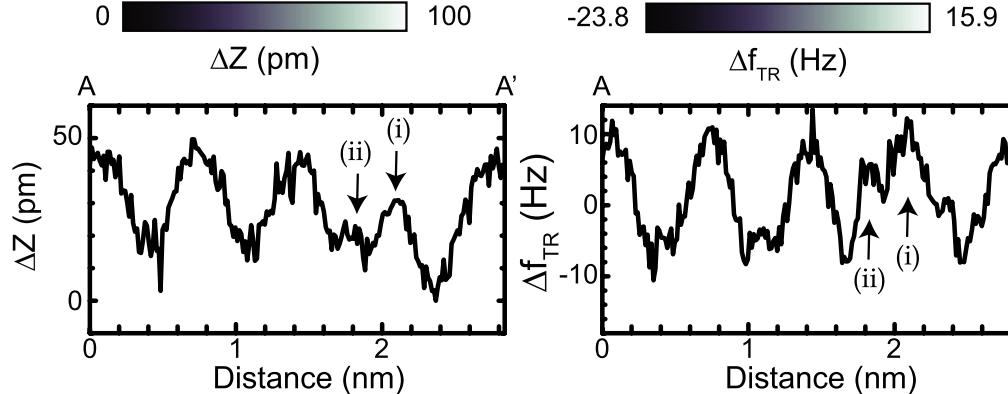

(c)
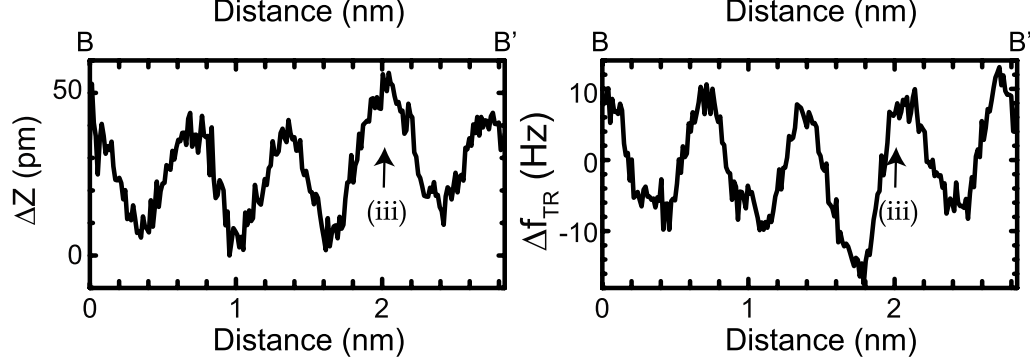

Figure 6. (a) Topographic image and $\Delta f_{T R}$ map of the dolomite surface with one carbonate group defect. (b), (c) Corresponding line profiles along $A-A^{\prime}$ and $B-B^{\prime}$, respectively. Measurement parameters: $\Delta f_{2 \text { nd }}=-100 \mathrm{~Hz}, f_{2 \text { nd }}=1020$ $010 \mathrm{~Hz}, A_{2 n d}=500 \mathrm{pm}$ and $Q_{2 n d}=13507 ; f_{\mathrm{TR}}=1519453 \mathrm{~Hz}, A_{\mathrm{TR}}=10 \mathrm{pm}$ and $Q_{\mathrm{TR}}=145568$.

and intensities can be distinguished along the [421direction. This feature was first observed on calcite (104) surfaces and is known as row-pairing reconstruction [53-55, 25]. In this reconstruction, spots with different intensities can be either perfectly aligned along the $\left[421^{-}\right]$direction or arranged in a zig-zag fashion (e.g. figures $5(\mathrm{a})$ and (e)). While both patterns can be mainly attributed to the interactions between the tip apex and the protruding oxygens of the carbonate groups on the surface, similar to the friction force microscopy images reported in [56], they are strongly dependent on the imaging conditions [25]. Furthermore, both the intensity and shape of the spots in topographic and $\Delta f_{T R}$ maps can be locally perturbed. This can be seen in figures $5(\mathrm{c})$ and (d), where 'interstitial' spots are resolved as indicated with arrows. Such perturbations of the spot patterns can be related to variations in the $\mathrm{Ca}-$ $\mathrm{Mg}$ distribution in the cation sublattice or to the presence of impurities.

Another kind of defect is shown in figure 6(a). Since the imaging contrast is associated with the row-pairing reconstruction, it seems that one carbonate group is missing in this case. At the position slightly offset to the defect site corresponding to the $\mathrm{Mg}$ and $\mathrm{Ca}$ atom sites, two small spots were observed both in the topographic and the $\Delta f_{\mathrm{TR}}$ map as indicated with arrows ((i) and (ii)). The corresponding line profiles along $A-A^{\prime}$ clearly show the differences of the corrugation amplitude and $\Delta f_{T R}$ (figure $6(b)$ ). The corrugation height of spot (i) is higher than that of spot (ii) and the $\Delta f_{\mathrm{TR}}$ of spot (i) is more positive than that of spot (ii). For the bimodal detection, the $\Delta f_{T R}$ is proportional to the lateral force gradient time averaged over the vertical oscillation amplitude. And for the attractive force region, the atomic site with a more attractive site, namely observed atoms in the topograph, gives rise to a more positive $\Delta f_{\mathrm{TR}}[57,20$, 21]. Therefore, this result shows clear evidence that the strength of the interaction at spot (i) is stronger than that at spot (ii). These different intensities might be related to the different magnitudes of the tip-sample interaction, arising from $\mathrm{Mg}$ and $\mathrm{Ca}$ atoms. Further, the carbonate group beside the defect (iii) was pronounced by $\approx 15 \mathrm{pm}$ in the topograph as shown in the line profiles

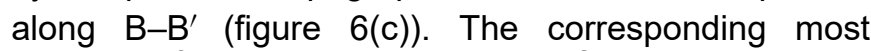
positive $\Delta f_{\mathrm{TR}}$ position is laterally shifted to the right by $\approx 100 \mathrm{pm}$. On the basis of this observed image contrast, the displacement of the carbonate group from the original position by the tip-sample interaction most probably plays a role in the row-pairing reconstruction in imaging. Systematic distance-dependent measurements of the interaction force and a corroboration via theoretical calculations can shed light on both the effect of cationic ordering and the presence of 
impurities and vacancies on the atomic-scale imaging $[58,46,51,59,60,47]$. Unfortunately, at RT a stable distance dependence of $\Delta f$ could not be measured on this dolomite sample due to an infrequent change of the tip condition. Measurements at cryogenic temperature would be thus very helpful for a deeper characterization of the dolomite (104) surface.

\section{Conclusion}

The morphology and stability of a mineral dolomite (104) surface were systematically studied by bimodal dynamic force microscopy for detection of the vertical and lateral tip-sample interactions with atomic resolution. We found that decomposition of the surface takes place by release of $\mathrm{CO}_{2}$ gas even at room temperature in an ultra-high vacuum so that the surface is not energetically stable. This instability might be related to the anomalous reactivity of dolomite (104) surfaces [11, 10, 12]. Such instability leads to the degradation of the surface before the measurement and makes a clean surface for atomic-scale imaging difficult to prepare. With a carefully chosen preparation procedure, namely cleaving in a vacuum and mild annealing, we have succeeded in obtaining an atomically clean surface. The main imaging contrasts arose from the carbonate groups. Dynamic force microscopy in an ultra-high vacuum for atomic-scale measurements has mainly been conducted with surfaces prepared in the laboratory. Now, this technique is becoming critical for studying surfaces of abundant terrestrial and extraterrestrial minerals [61] with high resolution.

\section{Acknowledgments}

This work was supported in part by the Swiss National Science Foundation, the Commission for Technology and Innovation CTI, the ESF EUROCORE programme FANAS and by the NCCR 'Nanoscale Science' of the Swiss National Science Foundation.

\section{References}

[1] Vaughan D and Pattrick R A D (ed) 1995 Mineral Surfaces

[2] Ohnesorge F and Binnig G 1993 Science 2601451

[3] Pina C M, Pimentel C and Garc'ia-Merino M 2010 Surf. Sci. 604

[4] de Boer R 1977 Geochim. Cosmochim. Acta 41265 [5] Arvidson R S and Mackenzie F T 1996 Aqua. Geochem. 273 [6] Reeder R J 1982 Estudios Geol'ogicos 38179

[7] Friedman G M 1973 Sedimentary Carbonate Minerals (Berlin: Springer) p 228

[8] Pokrovsky O S and Schott J 2001 Am. J. Sci. 301597

[9] Leeuw N H D 2002 Am. Mineral. 87679

[10] Higgins S R and Hu X 2005 Geochim. Cosmochim. Acta 692085

[11] Hu X, Grossie D A and Higgins S R 2005 Am. Mineral. 90 963 [12] Fenter P, Zhang Z, Park C, Sturchio N, Hu X and Higgins S 2007 Geochim. Cosmochim. Acta 71566

[13] Morita S, Wiesendanger R and Meyer E 2002 Noncontact Atomic Force Microscopy (Berlin: Springer)
[14] Morita S, Giessibl F J and Wiesendanger R 2009 Noncontact Atomic Force Microscopy vol 2 (Berlin: Springer)

[15] Albrecht T R, Gr"utter P, Horne D and Rugar D 1991 Phys. $69668 \quad$ J. Appl.

[16] Giessibl F J 1995 Science 26768

[17] Rodr'ıguez T R and Garc'ia R 2004 Appl. Phys. Lett. 84449 [18] Lozano J R and Garc'ia R 2008 Phys. Rev. Lett. 100076102 [19] Kawai S, Glatzel T, Koch S, Such B, Baratoff A and Meyer E 2009 Phys. Rev. Lett. 103220801

[20] Kawai S, Glatzel T, Koch S, Such B, Baratoff A and Meyer E 2010 Phys. Rev. B 81085420

[21] Kawai S, Pawlak R, Glatzel T and Meyer E 2011 Phys.

Rev. B 84085429

[22] Pfeiffer O, Bennewitz R, Baratoff A, Meyer E

and Grüutter P 2002 Phys. Rev. B 65161403

[23] Kawai S, Kitamura S, Kobayashi D and Kawakatsu $\mathrm{H}$ 2005 Appl. Phys. Lett. 87173105

[24] Kawai S, Canova F F, Glatzel T, Hynninen T, Meyer E and Foster A S 2012 Phys. Rev. Lett. 109146101

[25] Rahe P, Sch"utte1 J and K"uhnle A 2012 J. Phys.: Condens. Matter 24084006

[26] Sch "utte J, Rahe P, Tr"oger L, Rode S, Bechstein R, Reichling M and K"uhnle A 2010 Langmuir 268295 [27]

Howald L, Meyer E, L"uthi R, Haefke H, Overney R, Rudin H and G"untherodt H J 1993 Appl. Phys. Lett.

63117 [28] Kawai S, Kitamura S, Kobayashi D, Meguro S and Kawakatsu H 2005 Appl. Phys. Lett. 86193107

[29] Kawai S, Rose F, Ishii T and Kawakatsu H 2006 J. Appl. Phys. 99104312

[30] Stipp S, Gutmannsbauer W and Lehmann T 1996 Am. Mineral. 811

[31] Sommerhalter C, Matthes T W, Glatzel T, J"ager-

Waldau A and Lu x-Steiner M C 1999 Appl. Phys. Lett. 75286

[32] Rosenwaks Y, Shikler R, Glatzel T and Sadewasser S 2004 Phys. Rev. B $700 \quad 85320$

[33] Barth C and Henry C R 2007 Phys. Rev. Lett. 98

136804 [34] Sadewasser S and Lux-Steiner M C 2003

Phys. Rev. Lett. 91266101

[35] Bandi W and Krapf G 1976 Thermochim. Acta 14

221 [36] Wiedemann H-G and Bayer G 1987

Thermochim. Acta 121479

[37] McIntosh R, Sharp J and Wilburn F 1990

Thermochim. Acta 65281

[38] Dollimore D, Dunn J, Lee Y and Penrod B 1994

Thermochim. Acta 237125

[39] L'vov B V 2002 Thermochim. Acta 3861

[40] L'vov B V 2002 Thermochim. Acta 389199

[41] L'vov B V and Ugolkov V L 2003 Thermochim. Acta 401

139 [42] Powell E K and Searcy A W 1978 J. Am. Ceram.

Soc. 61216 [43] Kobayashi K, Yamada H, Horiuchi T and

Matsushige K 1999 Appl. Surf. Sci. 140281

[44] “Ozg"ur "Ozer H, O'Brien S J and Pethica J B 2007

Appl. Phys. Lett. 90133110

[45] Loske F, Rahe P and K"uhnle A 2009

2 Nanotechnology 20264010

[46] Schirmeisen A, Weiner D and Fuchs H 2006 Phys.

Rev. Lett.97 136101

[47] Kawai S, Glatzel T, Koch S, Baratoff A and Meyer E 2011 Phys. Rev. B 83035421

[48] Foster A S, Barth C, Shluger A L and Reichling M 2001

Phys. Rev. Lett. 862373

[49] Hoffmann R, Weiner D, Schirmeisen A and Foster A

S 2009 Phys. Rev. B 80115426

[50] P'erez R, Payne M C, Stich I and Terakura K 1997 Phys. Rev. Lett. 78678

[51] Sugimoto Y, Pou P, Abe M, Jelinek P, P'erez R, Morita S and Custance O 2007 Nature 44664 
[52] Teobaldi G, L“ammle K, Trevethan T, Watkins M, Schwarz A, Wiesendanger R and Shluger A L 2011 Phys. Rev. Lett. 106216102

[53] Rachlin A L, Henderson G S and Goh M C 1992 Am. Mineral.77 904

[54] Stipp S L and Hochella M F 1991 Geochim. Cosmochim. Acta $55 \quad 1723$

[55] Rode S, Oyabu N, Kobayashi K, Yamada H and

$$
\text { K"uhnle A } 2009 \text { Langmuir } 252850
$$

[56] Pina C M, Miranda R and Gnecco E 2012 Phys. Rev. B 85073402
[57] Kawai S, Sasaki N and Kawakatsu H 2009 Phys. Rev. B 79195412

[58] Lantz M A, Hug H J, Hoffmann R, van Schendel P J A Kappenberger P, Martin S, Baratoff 'A and

G"untherodt H J 2001 Science 2912580

[59] Albers B J, Schwendemann T C, Baykara M Z, Pilet N, Liebmann M, Altman E I and Schwarz U D 2009

Nature Nanotechnol. 4307

[60] Gross L, Mohn F, Moll N, Liljeroth P and Meyer G 2009 Science 3251110

[61] Nakamura T et al 2011 Science 3336046 